\title{
Amorphous nucleation precursor in highly nonequilibrium fluids
}

\author{
Gyula I. Tóth, ${ }^{1}$ Tamás Pusztai, ${ }^{1}$ György Tegze,${ }^{1}$ Gergely Tóth,${ }^{2}$ and László Gránásy ${ }^{1,3}$ \\ ${ }^{1}$ Research Institute for Solid State Physics and Optics, P. O. Box 49, H-1525 Budapest, Hungary \\ ${ }^{2}$ Institute of Chemistry, Eötvös University, P. O. Box 32, H-1518 Budapest, Hungary \\ ${ }^{3} B C A S T$, Brunel University, Uxbridge, Middlesex, UB8 3PH, UK
}

(Dated: June 7, 2021)

\begin{abstract}
Dynamical density functional simulations reveal structural aspects of crystal nucleation in undercooled liquids: the first appearing solid is amorphous, which promotes the nucleation of bcc crystals, but suppresses the appearance of the fcc and hcp phases. These findings are associated with features of the effective interaction potential deduced from the amorphous structure.

PACS numbers: 64.60.Q-, 64.60.My, 64.70.D-, 68.08.-p, 82.60.Nh
\end{abstract}

Mounting evidence indicates that the classical picture of crystal nucleation, which considers "heterophase" fluctuations of only the stable phase, is oversimplified. Early analysis by Alexander and McTague suggests preference for bcc freezing in simple liquids [1. Atomistic simulations for the Lennard-Jones (LJ) system have verified that small heterophase fluctuations have the metastable (MS) bcc structure, and even larger clusters of the stable (S) fcc structure have a bcc interface layer [2, while the ratio of the two phases can be tuned by changing the pressure 3. Composite bcc/fcc nuclei have also been predicted by continuum models [4. Two-stage nucleation has been reported in systems that have a metastable critical point in the undercooled liquid (including solutions of globular proteins [5] and eutectic alloys [6]); the appearance of the crystalline phase is assisted by liquid droplets, whose formation precedes/helps crystal nucleation [7]. Recent studies indicate a similar behavior in simple liquids such as the LJ [8] or hard-sphere (HS) 9] fluids, where dense liquid/amorphous precursor assists crystal nucleation. Analogous behavior has been reported for colloidal systems in 2D [10] and 3D [1]. Brownian Dynamics studies for the HS system [12] show the evolution of medium range crystalline order during the prenucleation stages. Liquid mediated crystal-amorphous and crystal-crystal transitions have also been predicted 13. These findings imply that the nucleation precursors are fairly common. A deeper understanding of nucleation pathways requires a systematic study of a system, in which amorphous and crystalline structures compete during solidification.

Such a system is defined by the single-mode phase-field crystal (1M-PFC) model of Elder et al. [14, a simple dynamical density functional theory, which has bcc, fcc, and hcp stability domains [15, and the appearance of amorphous phase and two-step nucleation has also been reported [16]. A two-mode extension of the model by $\mathrm{Wu}$ et al. (2M-PFC) has been designed to promote fcc solidification [17, whereas with a specific choice of model parameters the 1M-PFC model can also be recovered.

Herein, we address crystal nucleation in PFC models interpolating between the 1M-PFC and 2M-PFC limits.

First, we re-cast the free energy of the PFC models in terms of $\lambda=R_{1} /\left(1+R_{1}\right) \in[0,1]$ ( $R_{1}$ is the relative strength of the first- and second-mode contributions [17]), a parameter that can be used to interpolate between the 2M-PFC $(\lambda=0)$ and 1M-PFC $(\lambda=1)$ models:

$$
\begin{aligned}
& \mathcal{F}=\int d \mathbf{r}\left\{\frac { \psi } { 2 } \left[\epsilon+\left(1+\nabla^{2}\right)^{2}\right.\right. \\
& \left.\left.\times\left(\lambda+\{1-\lambda\}\left\{Q_{1}^{2}+\nabla^{2}\right\}^{2}\right)\right] \psi+\frac{\psi^{4}}{4}\right\},
\end{aligned}
$$

where $\psi \propto\left(\rho-\rho_{L}^{r e f}\right) / \rho_{L}^{r e f}$ is the scaled density difference relative to the reference liquid of particle density $\rho_{L}^{r e f}$. The reduced temperature $\epsilon$ can be related to the bulk moduli of the fluid and the crystal, whereas $Q_{1}=q_{1} / q_{0}$ $\left(=2 / 3^{1 / 2}\right.$ for fcc [17]) is the ratio of the wave numbers corresponding to the two modes.

The respective dimensionless Euler-Lagrange equation (ELE) and equation of motion (EOM) read as $\frac{\delta \mathcal{F}}{\delta \psi}=$ $\left(\frac{\delta \mathcal{F}}{\delta \psi}\right)_{\psi_{0}}$, and $\frac{\partial \psi}{\partial \tau}=\nabla^{2} \frac{\delta \mathcal{F}}{\delta \psi}+\zeta$, respectively, where $\frac{\delta \mathcal{F}}{\delta \psi}$ denotes the functional derivative of $\mathcal{F}$ with respect to $\psi$, and $\tau$ is the dimensionless time. The RHS of the ELE is taken at the far-field value $\psi_{0}$ (homogeneous liquid). In the EOM, the fluctuations are represented by a colored Gaussian noise $\zeta$ of correlator $\left\langle\zeta(\mathbf{r}, \tau) \zeta\left(\mathbf{r}^{\prime}, \tau^{\prime}\right)\right\rangle=$ $-\alpha \nabla^{2} g\left(\left|\mathbf{r}-\mathbf{r}^{\prime}\right|, \sigma\right) \delta\left(\tau-\tau^{\prime}\right)$, where $\alpha$ is the noise strength, and $g\left(\left|\mathbf{r}-\mathbf{r}^{\prime}\right|, \sigma\right)$ a high frequency cutoff function [18] for wavelengths shorter than the interatomic spacing $(\sigma)$. Due to the overdamped conservative dynamics the EOM realizes, the PFC models defined so are suitable for describing crystalline colloidal aggregation [19, 20]. These equations have been solved numerically [21] on rectangular grids of typical size of $512 \times 256 \times 256$ (ELE) and $256 \times 256 \times 256(\mathrm{EOM})$, assuming a periodic boundary condition. The ELE has been used to determine the phase diagram, the driving force, the nucleation barrier, 

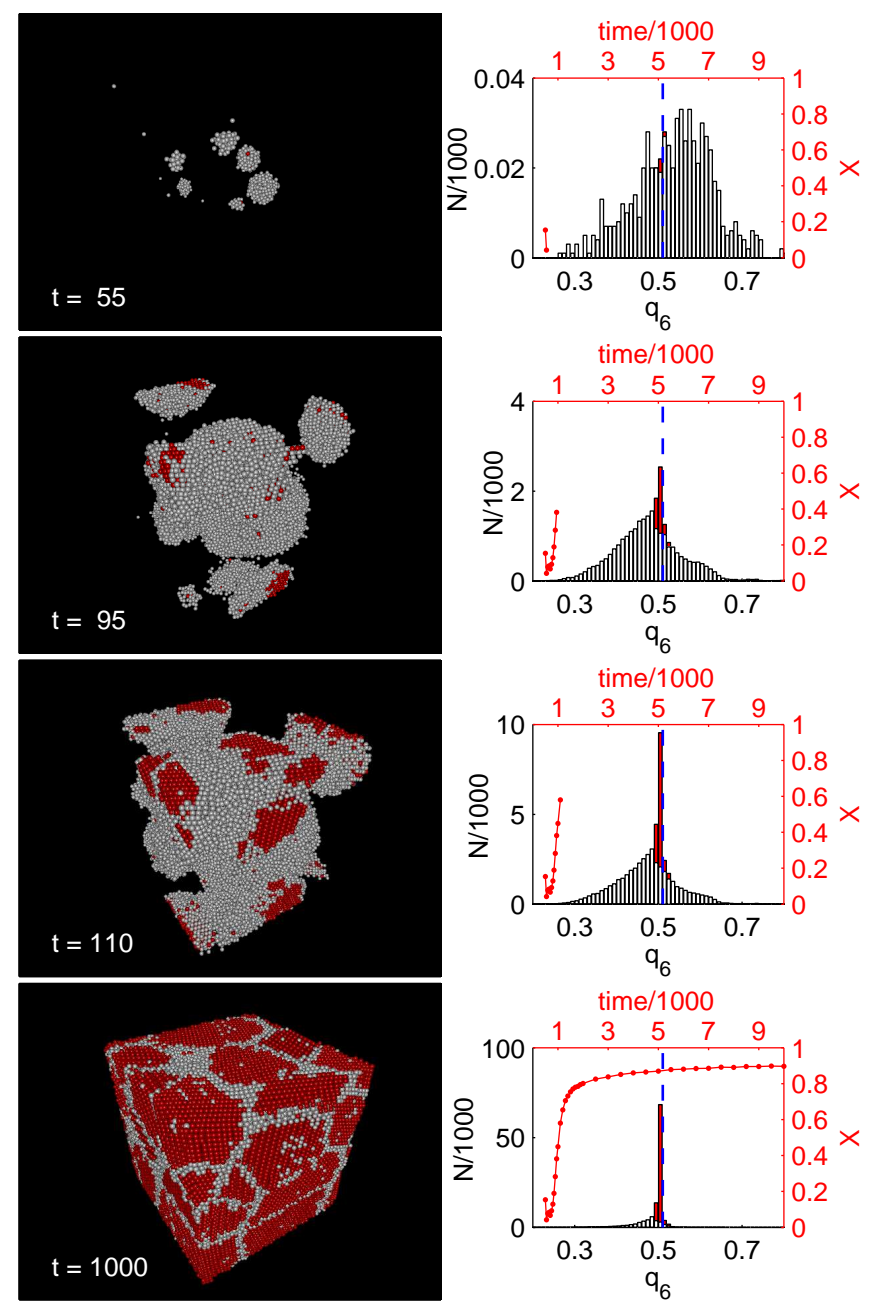

FIG. 1: (color online) Two-step nucleation in the 1M-PFC model at $\epsilon=-0.1667$ and $\psi_{0}=-0.25$. Left: Snapshots of the density distribution taken at the dimensionless times $\tau=57.74 t$. Spheres of diameter of the interparticle distance centered on density peaks higher than a threshold (0.15) are shown that are colored red if $q_{4} \in[0.02,0.07]$ and $q_{6} \in[0.48,0.52]$ (bcc-like), and white otherwise. Right: population distribution of $q_{6}$ (histogram painted similarly), and the time-dependence of the fraction $X$ of bcc-like neighborhoods (solid line). Note the nucleation of amorphous clusters and the formation of amorphous grain boundaries 23 .

and the coexistence properties, including the densities and the solid-liquid interface free energy (as described in [15]), while the EOM has been applied to simulate nucleation. Owing to the effect of noise on the free energy, the results from the two approaches converge for $\zeta \rightarrow 0$.

The results of the nucleation studies performed solving the EOM under condition described in 22] are summarized in Figs. 1 and 2, In the case of the 1M-PFC model, in a large part of the bcc stability domain we have observed two-step nucleation starting with formation of amorphous clusters, in which the bcc phase nucleates subsequently (see Fig. 11). We have used the $q_{4}$
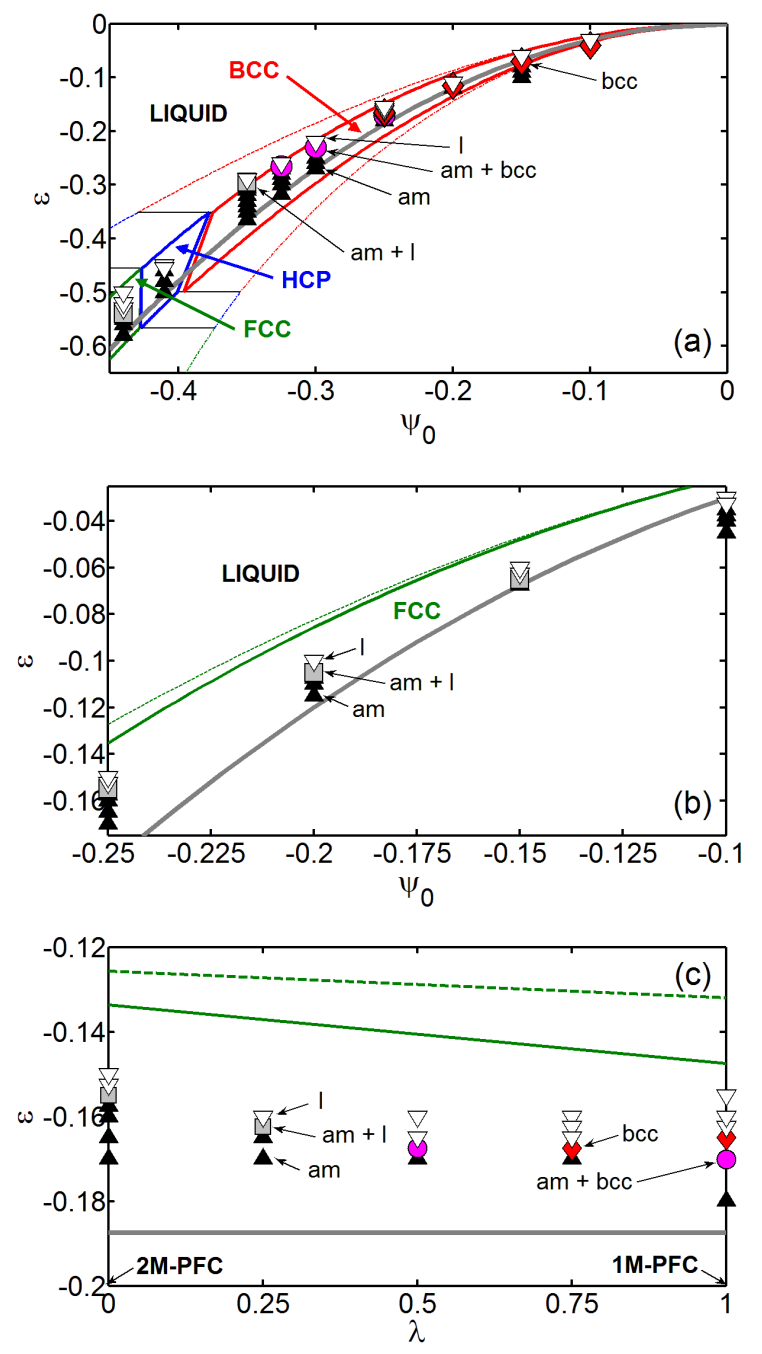

FIG. 2: (color online) Nucleation map for PFC models: (a) 1M-PFC; (b) 2M-PFC; (c) dependence on $\lambda$ at $\psi_{0}=-0.25$. The phase content obtained after $10^{5}$ time steps is shown: open triangle - liquid; square - (amorphous + liquid); circle (amorphous + bcc); diamond - bcc; full triangle-amorphous. The heavy grey line stands for the stability limit of the liquid. Parts of the phase diagram are also shown.

and $q_{6}$ order parameter to characterize the local structure [2]. With increasing undercooling, the nucleation rate of the amorphous clusters increases, leading to spatially nearly homogeneous transition at high undercoolings. In contrast, we have not detected any phase transition for more than $10^{6}$ time steps at $\epsilon=-0.1598$. These findings strongly indicate that crystal nucleation is enhanced by the amorphous precursor, and that direct bcc crystal nucleation from the liquid requires orders of magnitude longer time than via the precursor. This behavior appears analogous to the role the non-crystalline precursor plays in colloids [10, 11] and simple liquids [8, 9].

We were unable to nucleate crystalline phases other than bcc in the 1M-PFC model [Fig. 2(a)]. Even in the 

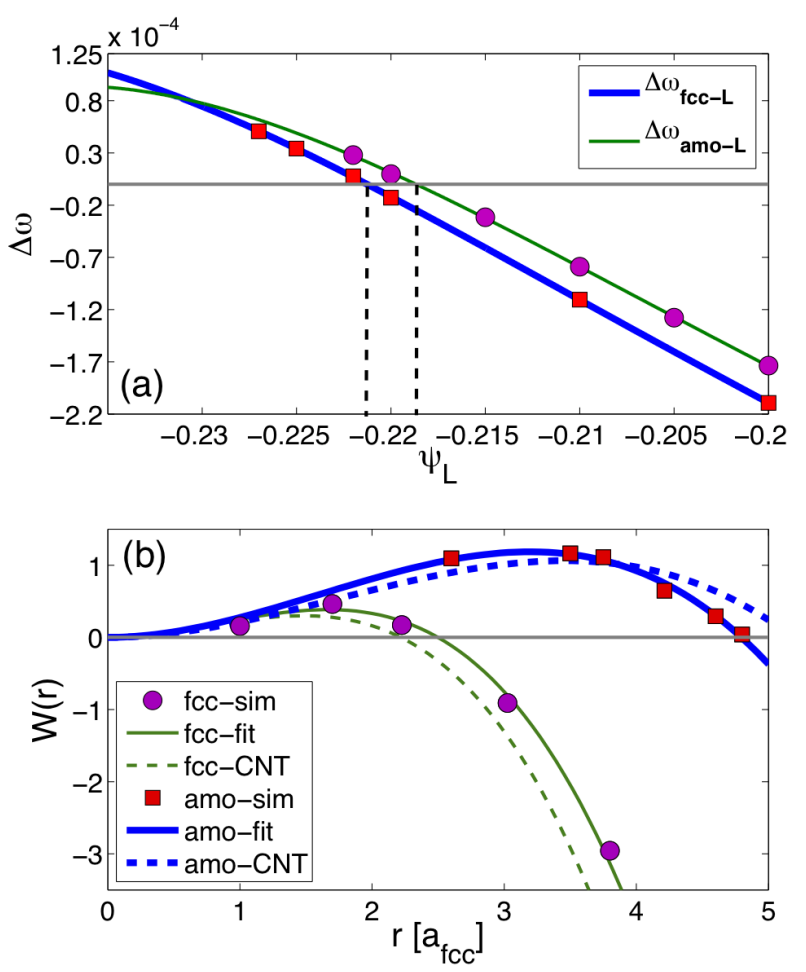

FIG. 3: (color online) Properties of the amorphous phase in the 2M-PFC limit from the ELE at $\epsilon=-0.1$ : (a) Driving force for the liquid to amorphous transition $\left(\Delta \omega_{a m o-L}\right)$. For comparison the driving force for fcc freezing $\left(\Delta \omega_{f c c-L}\right)$ is also shown. The vertical lines indicate liquid densities at the (S) fcc-liquid and (MS) amorphous-liquid coexistences. (b) Nucleation barrier for the amorphous and fcc phases at $\psi_{0}=$ -0.21775 . ( $a_{f c c}$ - lattice constant of the fcc structure.) Fits of $W=A r^{3}+B r^{2}$ (solid lines) are compared with estimates from the classical droplet model using the equilibrium interface free energy and the driving force (dashed lines).

stability domain of the hcp and fcc phases, the amorphous phase formed in the time window of the simulations. Remarkably, this stayed so even in the 2M-PFC limit [Fig. 2(b)]. Interestingly, the amorphous phase appears to coexist with the liquid, indicating a first-order phase transition between these phase, in agreement with the observed nucleation of the amorphous state. This suggests significant differences (e.g., in density) between the liquid and amorphous phases. Varying $\lambda$ at $\epsilon=-0.1$, we see a gradual transition from the 1M-PFC behavior (liquid $\rightarrow$ amorphous $\rightarrow$ bcc) to the behavior seen on the 2M-PFC side (liquid $\rightarrow$ amorphous) [Fig. 22(c)]. (Comparable results were obtained for constant cooling rates.)

To investigate the lack of fcc crystallization in the 2MPFC model specifically designed to crystallize to the fcc phase, we used the ELE at $\epsilon=-0.1$ for determining the free energy $\gamma$ of the fcc-liquid and amorphous-liquid interfaces (Table I), and the driving force $\Delta \omega$ for fcc freezing and amorphization [Fig. 3(a)] (see the methodology in [15]), whose interplay determines the nucleation rate [24.
In the density range of interest the fcc phase is preferred thermodynamically [Fig. 3(a)]. Between the fcc-liquid and amorphous-liquid coexistences, there is no driving force for amorphization, so fcc freezing should take place. To evaluate $\gamma$, we have created equilibrium sandwiches (fcc-liquid-fcc and amorphous-liquid-amorphous), solved the ELE, and determined the grand potential emerging from the two interfaces. Comparable density changes were found at the amorphous-liquid and fcc-liquid transitions (Table I), a finding consistent with the first order amorphization transition implied earlier. Remarkably, $\gamma_{a m-L}^{e q} \approx 0.67 \gamma_{f c c-L}^{e q}$ (Table I). The nucleation barriers calculated for $\psi_{0}=-0.21775$ using the classical droplet model, $W(r)=(4 \pi / 3) r^{3} \Delta \omega+4 \pi r^{2} \gamma$, are shown in [Fig. 3(b)]. At this supersaturation fcc nucleation is clearly preferable. Yet, we have not seen freezing even after $1.9 \times 10^{7}$ time steps. The liquid density beyond which $W_{f c c}>W_{a m}$ is $\psi_{0} \approx-0.21$. This is consistent with the finding that in the density range, where solidification could be observed at all $\left(\psi_{0} \geq-0.1962\right.$ for $10^{7}$ time steps), the amorphous phase nucleated. It appears that we cannot observe fcc nucleation because of a technical difficulty: the time accessible for simulations is too short. Note that the dynamic EOM studies and the equilibrium ELE results consistently indicate separate time scales for changes of density and structure.

For the PFC models the free energy barrier has a rough surface with many local minima that can be mapped out directly by using the ELE [15. This provides an independent test of the previous computations. The results shown in [Fig. 3(c)] indicate a reasonable agreement between the predicted (dashed lines) and the directly evaluated barriers (symbols + solid lines). This is reflected in the similarity of the interfacial properties evaluated in equilibrium and from fitting the droplet model (see Table I). The minor discrepancy presumably originates from the fact that for such nanoclusters the classical droplet model is probably not very accurate.

To rationalize the dominance of amorphous solidification in a substantial part of the phase diagram, we have evaluated effective pair potentials for the $1 \mathrm{M}$ - and $2 \mathrm{M}$ PFC models from the pair correlation function of the respective amorphous phases [Fig. 4(a)] using Schommers' iterative method 25 that works reasonably for single component systems 26. The potentials obtained are similar for short distances, and have a peak at $\sim r_{0} \sqrt{2}$,

TABLE I: Equilibrium densities $\left(\psi^{e q}\right)$ and interface free energies $(\gamma)$ in equilibrium (eq) and from fitting to $W(r)$ (fit) for the 2M-PFC model at $\epsilon=-0.1$.

\begin{tabular}{ccccc}
\hline \hline $\mathrm{X}$ & $\psi_{L}^{e q}$ & $\psi_{S}^{e q}$ & $\gamma_{X-L}^{e q}$ & $\gamma_{X-L}^{\text {fit }}$ \\
\hline $\mathrm{am}$ & -0.21885 & -0.21404 & $1.79 \times 10^{-4}$ & $2.34 \times 10^{-4}$ \\
$\mathrm{fcc}$ & -0.22139 & -0.21629 & $2.76 \times 10^{-4}$ & $2.80 \times 10^{-4}$ \\
\hline \hline
\end{tabular}

Subscripts $\mathrm{S}$ and L stand for solid and liquid, respectively. 

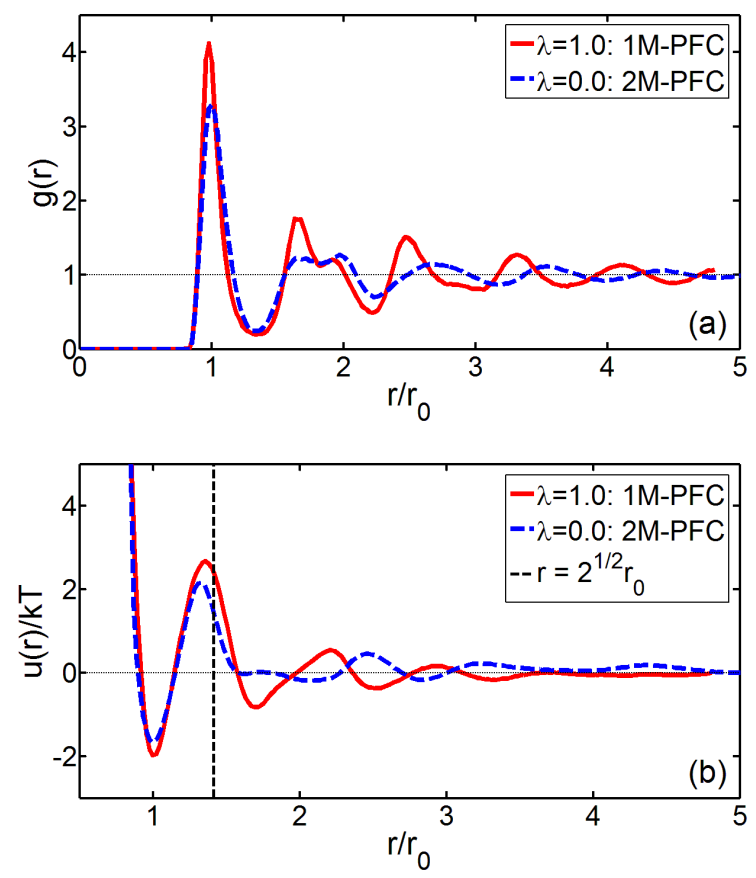

FIG. 4: (color online) Amorphous structure and effective pair potential in the 1M- and 2M-PFC models: (a) $g(r)$ of the amorphous structures. (b) Effective pair potentials evaluated from $g(r)$ by Schommers' method.

where $r_{0}$ is the radius at the main minimum of the potential [Fig. 4(b)]. Remarkably, such potentials have been designed to realize monatomic glassformers, as a peak at $r_{0} \sqrt{2}$ suppresses the close packed crystal structures [27. Hence, we associate the evident difficulty to produce the fcc and hcp phases with this feature of the PFC effective interaction potentials. Furthermore, in the presence of multiple minima of the interaction potential coexistence of disordered phases is expected [28, as indeed seen here.

Summarizing, the PFC models display MS amorphousliquid coexistence and first-order amorphization. In the cases accessible for dynamic simulations, the nucleation of the amorphous phase is faster than crystal nucleation. This leads to a separation of time scales for density and structural changes, as seen in other systems [9. However, some details differ: Such coexistence is unknown in the HS system, while the fcc and hcp structures are suppressed here. It is also unclear whether along the reaction coordinate specified in Ref. 8, the free energy landscape of the PFC models is similar to that of the LJ system.

Combining the results obtained for various potentials (LJ, HS, the present PFC potentials, etc. [8, 9, 27, 28]), it appears that a repulsive core suffices for the appearance of a disordered precursor, whereas the peak at $\sim r_{0} \sqrt{2}$ correlates with the observed suppression of fcc and hcp structures, while the coexistence of the liquid and amorphous phases seen here can be associated with multiple minima of the interaction potential.
This work was supported by the EU FP7 Project ENSEMBLE under Grant Agreement NMP4-SL-2008213669 and by TAMOP 4.2.1B-09/1/KMR-2010-0003.

[1] S. Alexander and J. McTague, Phys. Rev. Lett. 41, 702 (1978).

[2] P. R. ten Wolde, M.J. Ruiz-Montero, and D. Frenkel, Phys. Rev. Lett. 75, 2714 (1995).

[3] C. Desgranges and J. Delhommelle, Phys. Rev. Lett. 98, 235502 (2007); J. Am. Chem. Soc. 128, 10368 (2006).

[4] Y. C. Shen and D. W. Oxtoby, Phys. Rev. Lett. 77, 3585 (1996); G. I. Tóth, J. R. Morris, and L. Gránásy, Phys. Rev. Lett. 106, 045701 (2011).

[5] O. Galkin and P. G. Vekilov, Proc. Natl. Acad. Sci. U.S.A. 97, 6277 (2000); W. Pan, A. B. Kolomeisky, and P. G. Vekilov, J. Chem. Phys. 122, 174905 (2005).

[6] G. I. Tóth and L. Gránásy, J. Chem. Phys. 127, 074710 (2007).

[7] P. R. ten Wolde and D. Frenkel, Science 277, 1975 (1997); V. Talanquer and D. W. Oxtoby, J. Chem. Phys. 109, 223 (1998).

[8] J. F. Lutsko and G. Nicolis, Phys. Rev. Lett. 96, 046102 (2006).

[9] T. Schilling et al., Phys. Rev. Lett. 105, 025701 (2010).

[10] T. H. Zhang and X. Y. Liu, J. Am. Chem. Soc. 129, 13520 (2007); J. Phys. Chem. B 111, 14001 (2007).

[11] H. J. Schöpe, G. Bryant, and W. van Megen, Phys. Rev. Lett. 96, 175701 (2009).

[12] T. Kawasaki and H. Tanaka, Proc. Natl. Acad. Sci. U. S. A. 107, 14036 (2010).

[13] V. I. Levitas, Phys. Rev. Lett. 95, 075701 (2005).

[14] K. R. Elder, M. Katakowski, M. Haataja, and M. Grant, Phys. Rev. Lett. 88, 245701 (2002).

[15] G. Tegze et al., Phys. Rev. Lett. 103, 035702 (2009); A. Jaatinen and T. Ala-Nissila, J. Phys.: Condens. Matter 22, 205402 (2010); G. I. Tóth et al. J. Phys.: Condens. Matter 22, 364101 (2010).

[16] J. Berry, K. R. Elder, and M. Grant, Phys. Rev. E 77, 061506 (2008).

[17] K.-A. Wu, A. Adland, and A. Karma, Phys. Rev. E 81, 061601 (2010).

[18] J. G. Ojalvo and J. M. Sancho, Noise in Spatially Extended Systems (Springer, 1999) p. 175

[19] S. van Teeffelen et al., Phys. Rev. B 79, 051404 (2009).

[20] G. Tegze et al., Soft Matter 7, 1789 (2011); G. Tegze, G. I. Tóth, and L. Gránásy, Phys. Rev. Lett. 106, 195502 (2011).

[21] G. Tegze et al. J. Comput. Phys. 228, 1612 (2009).

[22] $\Delta x=1.0 ; \Delta \tau=0.5774 ; \alpha=0.42$; the number of time steps was $10^{5}$, if not stated otherwise. The simulations started with a homogeneous liquid of density $\psi_{0}$.

[23] H. Zhang et al. Proc. Natl. Acad. Sci. U. S. A. 106, 7735 (2009).

[24] The nucleation prefactors are expected to be similar for crystallization and amorphization: both are determined by the molecular mobility in the liquid state.

[25] W. Schommers, Phys. Rev. A 28, 3599 (1983).

[26] G. Tóth, J. Phys.: Condens. Matter 19, 335220 (2007).

[27] J. P. K. Doye et al., J. Chem. Phys. 118, 2792 (2003).

[28] O. Mishima and H. E. Stanley, Nature 396, 329 (1998). 